\title{
МОДУЛЬНАЯ ЗАЖИМНАЯ СИСТЕМА ДЛЯ АВТОМАТИЗИРОВАННОЙ ЗАГРУЗКИ СТАНКА
}

\section{МаркУс МИХЕЛЬБЕРГЕР, Соня АУФРЕХТ}

\begin{abstract}
Представлены новинки компании SCHUNK в сфере зажимных систем и приспособлений. Отдельное внимание уделено перспективным интеллектуальным зажимным приспособлениям, способным осуществлять постоянный мониторинг прижимного усилия и положения фиксирующего ползуна за счет встроенных тензометрических и индуктивных датчиков, измерителей усилия.
\end{abstract}

В настоящее время сохраняется курс на автоматизацию загрузки станков и обрабатывающих центров. Это также оказывает влияние на технологию зажима: технологическая оснастка все больше и больше приспосабливается к требованиям процессов, выполняемых без обслуживающего персонала. Вдобавок к надежности, доступности и гибкости технологического процесса также приобретает большое значение эффективность использования электроэнергии и времени цикла. Исследование технологий продвинулось еще дальше: «умные» зажимные системы будущего дадут возможность создать интеллектуальную сеть с обрабатывающими центрами и транспортно-загрузочными устройствами.

\section{ДОПОЛНИТЕЛЬНОЕ ВОЗДЕЙСТВИЕ НА \\ ВАРИАТИВНОСТЬ, ВРЕМЯ ЦИКЛА \\ И ЭФФЕКТИВНОСТЬ ИСПОЛЬЗОВАНИЯ ЭЛЕКТРОЭНЕРГИИ}

Например, для механизированного патрона ROTA NCO компания SCHUNK разработала специальную систему быстрой смены кулачков, позволяющую заменять кулачки вручную без использования инструмента менее чем за одну минуту, при этом повторяемость составляет до 0,02 мм. При использовании различных наборов стандартных кулачков и индивидуально сконфигурированных специальных кулачков можно покрыть значительную часть вариативности за счет автоматизи- рованной загрузки с минимальным временем наладки. Компания SCHUNK идет еще дальше и внедряет свои первые устройства с полностью автоматизированной сменой кулачков, благодаря чему можно обойтись без какого-либо вмешательства оператора. Теперь транспортно-загрузочное устройство берет на себя не только загрузку токарного патрона, но также и смену зажимных кулачков либо по принципу «один кулачок за другим», либо путем единовременной замены всего комплекта кулачков (что особенно эффективно).

B сверхлегком токарном патроне SCHUNK ROTA NCE используется иной подход: он адаптирован для максимальной эффективности в крупносерийном производстве, и также может загружаться автоматически. Один патрон сочетает в себе аспекты облегченной конструкции, максимальную нагрузочную способность и большое сквозное отверстие. По сравнению с обычными токарными патронами в зависимости от размера модели инерция была снижена на величину до 40\% (рис. 1). Следовательно, токарный патрон может ускоряться и замедляться гораздо быстрее, чем традиционные механизированные патроны. В частности, в крупносерийном производстве уменьшенная масса приводит к более короткому времени цикла и сниженным затратам энергии. К аналогичной стратегии компания SCHUNK прибегает и в своих механизированных патронах ROTA NCA со специальным уплотнением и минимальными затратами на обслуживание. 
Они также имеют уменьшенный вес и благодаря особой геометрии отличаются упрощенным доступом, особенно при использовании в современных токарно-фрезерных обрабатывающих центрах. Благодаря большому сквозному отверстию этот патрон с высокой эффективностью использования электроэнергии и времени цикла также идеально подходит для автоматизированной загрузки прутка.

\section{ВЫСОКАЯ ПОВТОРЯЕМОСТЬ ЗАЖИМА ВО ВРЕМЯ АВТОМАТИЗИРОВАННОГО ПРОЦЕССА}

В дополнение к механизированным патронам, особенно в условиях, где требуется высокая точность и присутствует кольцевое напряжение, в автоматизированной загрузке также имеют свои преимущества гидроразжимные оправки (например, при шлифовании, фрезеровании, прорезании пазов, точении и строгании, а также при балансировке, измерении и испытании). Например, тонкие гидроразжимные инструменты с запатентованным компанией SCHUNK осевым возвратом (рис. 2) представляют собой эффективное решение для сведения к минимуму времени наладки при высочайшей точности и экономии дополнительных расходов. Они позволяют максимально быстро отцентрировать и зажать заготовки с повторяемостью $<0,003$ мм. Кроме того, осевой возврат обеспечивает абсолютно плоскую рабочую поверхность, благодаря чему заготовка выталкивается на плоскую поверхность в осевом направлении с помощью мембраны. Это препятствует даже минимальному нарушению баланса трубы и обеспечивает высокую устойчивость во время механической обработки.

Благодаря гидроразжимной технологии, позволяющей точно выровнять деталь по центру оси, можно полностью автоматизировать процессы при кратчайшем времени цикла и без необходимости его измерения. По запросу могут быть поставлены токарные патроны или удлиняемые оправки, которые всегда изготавливаются на базе конкретной заготовки и оснащаются второй гидравлической передачей и воздушным соединением для контроля контакта.

Зажимные блоки с большим усилием зажима SCHUNK TANDEM plus специально разработаны для автоматизированной загрузки станка (рис. 3). Квадратное основание и компактные размеры тисков обеспечивают плотную компоновку, благодаря которой в любое время можно зажать и обработать максимально возможное количество заготовок. В версии с пружинным действием тиски идеально подходят для подготовки к многоместным зажимным стойкам и паллетам, для хранения в накопителе заготовок и в то же время - для загрузки в станок с целью обработки. Оптимизированный внешний контур,

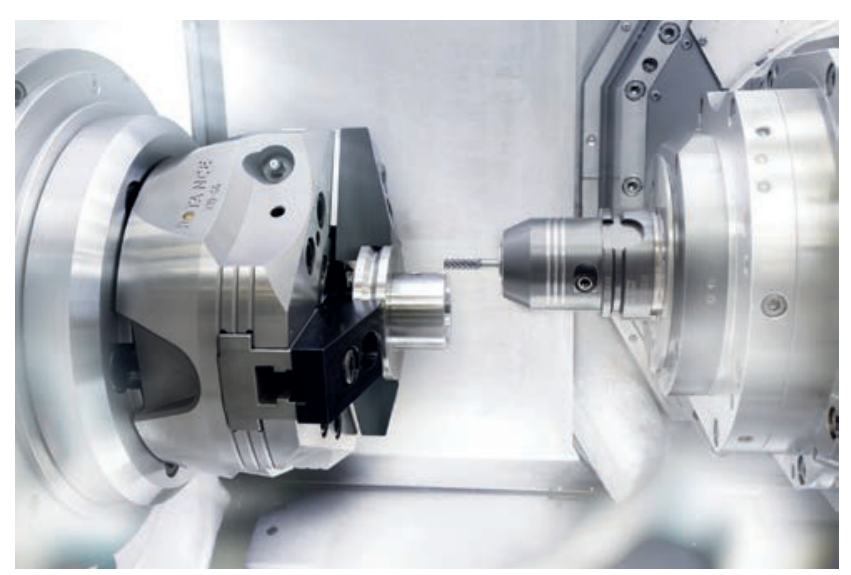

Puc. 1. Оптимизированная по весу конструкция токарного патрона позволяет снизить инерцию SCHUNK ROTA NCE на величину до 40\%. В частности, в серийном производстве токарный патрон являет собой впечатляющую демонстрацию высоких динамических характеристик, низкой себестоимости и эффективности использования электроэнергии

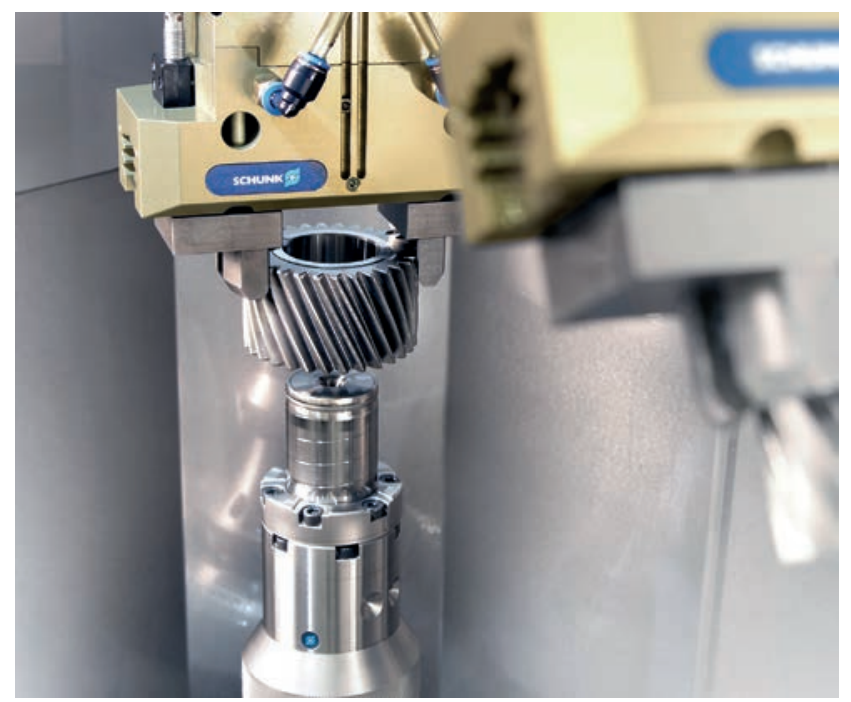

Puc. 2. Гидроразжимные оправки SCHUNK с функцией осевого прижатия эффективно оптимизируют время наладки при выполнении высокоточных работ по механической обработке. Их можно загружать автоматически и быстро заменить зажимное приспособление, не тратя времени на его выравнивание и привинчивание

специальная конструкция базовых кулачков, а также защитные планки, минимальный зазор и заглушки для крепежных винтов предотвращают образование пылевых карманов и попадание стружки и пыли в тиски с последующим оседанием. Во время автоматизированного процесса тиски можно акти- 


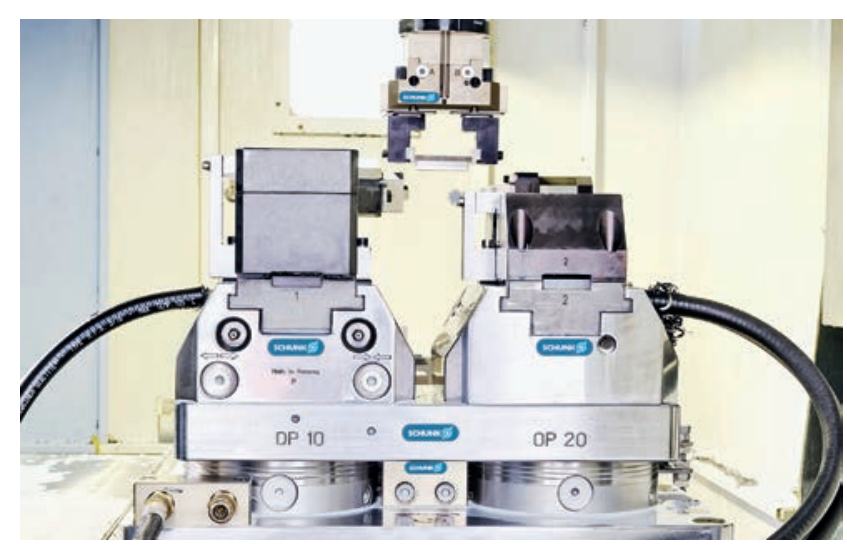

Puc. 3. Силовые зажимные блоки SCHUNK TANDEM plus с большим усилием зажима были специально разработаны для соблюдения требований по автоматизированной загрузке станка. В этом компактном решении по автоматизации они объединены с зажимной станцией SCHUNK VERO-S NSL3 и загружаются с помощью собственных осей станка

вировать пневматически, гидравлически или действием пружины, при этом повторяемость зажатия составляет до 0,01 мм. В стандартизированных версиях с длинным ходом также можно зажимать детали различных размеров или перекрывать мешающие контуры. Индуктивные бесконтактные датчики позволяют осуществлять непрерывный мониторинг технологического процесса.

\section{ЛЕГКИЕ В НАСТРОЙКЕ И КОМПАКТНЫЕ}

Даже если автоматизированная загрузка производится с помощью систем паллет и многоместных зажимных стоек, была доказана эффективность использования специально приспособленных механизированных зажимных систем. Они дают преимущества в части надежности технологического процесса, доступности и компактности, а также в том, что касается работы на станции установки. Например, ручные базовые зажимные тиски SCHUNK KONTEC KSC (рис. 4) представляют собой особенно эффективное универсальное устройство для механической обработки заготовок и готовых деталей с автоматическим накопителем заготовок.

Они сочетают в себе большое усилие зажима, удобство в эксплуатации и малое время наладки. При сравнительно небольших значениях крутящего момента они способны достичь усилия зажатия 50 кН (размер 160). Это означает, что отдельная гидравлическая штамповальная станция для зажима по форме больше не требуется. Поскольку зажим производится за счет натяжения, сводится к минимуму изгибающая нагрузка на корпус основания и,

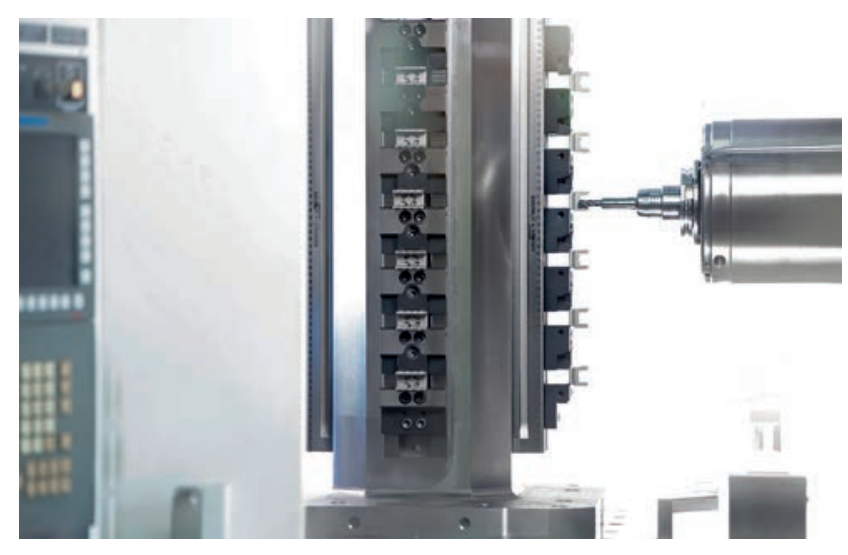

Puc. 4. Многоместные зажимные тиски SCHUNK KONTEC KSM обеспечивают множество решений по зажатию серийных деталей, при этом быстро и легко настраиваются

следовательно, подъем тисков, что дополнительно повышает точность и прочность зажатия. Центральный подшипник с предварительным натягом и без люфта реверсивного вращения шпинделя, а также специально отрегулированные направляющие скольжения гарантируют идеальную повторяемость, составляющую $\pm 0,015$ мм. Полностью герметизированный привод и встроенный отвод стружки обеспечивают особенно высокую стабильность технологического процесса и минимальный износ. Базовые зажимные тиски можно заказать в версии центрирующих зажимных тисков или тисков с прижимом к неподвижному кулачку. Они дают возможность быстро отрегулировать диапазон зажима, имеют плоскую конструкцию и малый вес - идеальные условия для транспортировки заготовок без обслуживающего персонала. Многоместные зажимные тиски SCHUNK KONTEC KSM2 также обладают преимуществом, заключающимся в зажатии нескольких заготовок рядом друг с другом в ограниченном пространстве. Тонкие кулачки патрона без выступающих мешающих контуров обеспечивают особенно высокую плотность установки заготовок и оптимальную доступность. Закаленная и тонко отшлифованная насечка гарантирует максимальную прочность, точность и стабильность формы. Независимо от размера заготовки в процессе зажима она автоматически прижимается при помощи клиновой зажимной системы. В результате обеспечивается безопасная и точная механическая обработка даже при высоких скоростях подачи. Нужен всего один ключ, чтобы зажать заготовки, полностью удалить кулачки с помощью системы быстрой смены кулачков или быстро и легко приспособить зажимную систему к различным заготовкам. Для этой цели предусмотрена широкая модульная система зажимных кулачков. 


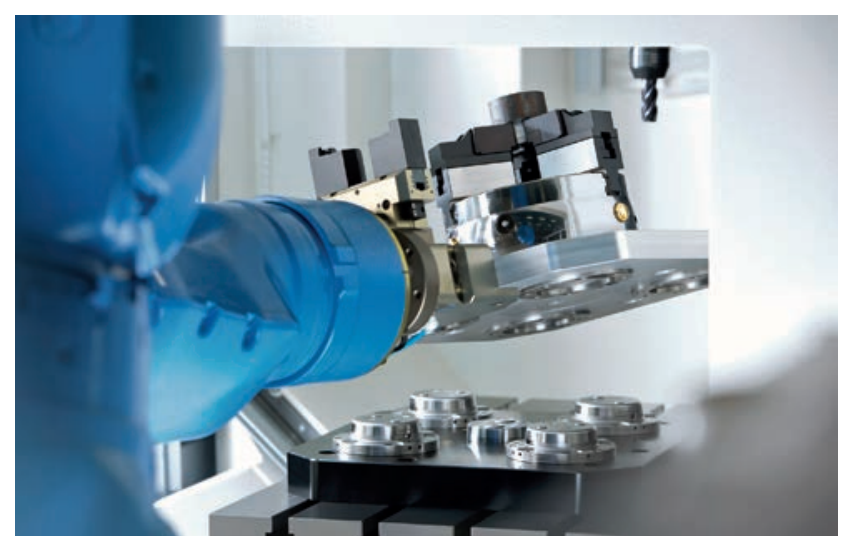

Puc. 5. При обработке малых и средних партий система быстрой смены паллет SCHUNK VERO-S NSA plus обеспечивает высокую надежность, гибкость и эффективность технологического процесса

\section{ТОНКИЕ СИСТЕМЫ БЫСТРОЙ СМЕНЫ ПАЛЛЕТ ДЛЯ} МАКСИМАЛЬНОЙ УНИВЕРСАЛЬНОСТИ

Такие стандартизированные системы быстрой смены паллет, как плоский модуль SCHUNK VERO-S NSA plus (рис. 5), гарантируют чрезвычайно гибкую замену зажимных приспособлений при стабильном технологическом процессе. В отличие от других зажимных систем, во время обработки она не требует подачи какой-либо энергии. Внутренний комплект пружин обеспечивает постоянное и безопасное удерживающее усилие даже при внезапном падении давления в пневматической системе. Благодаря запатентованной системе быстрого хода достигается прижимное усилие до $15000 \mathrm{H}$, при этом удерживающее усилие превышает 100000 Н. Все функциональные компоненты, например корпус, фиксирующие ползуны и зажимные кольца, изготовлены из закаленной нержавеющей стали и, следовательно, обладают абсолютной устойчивостью к коррозии. Во время автоматизированной смены нагнетаемый воздушный поток очищает плоские поверхности и короткий конус, тем самым обеспечивая отсутствие стружки и плоскую рабочую поверхность паллеты. Центрирующие поверхности гарантируют точное позиционирование в соответствии с базовой точкой. Фиксирующие ползуны обеспечивают самоблокирующуюся установку в соответствии с формой. Благодаря мониторингу динамического давления можно эффективно контролировать все этапы технологического процесса. В сочетании с тонкими легкими соединениями SCHUNK NSR с минимальными мешающими контурами можно производить смену паллет с чрезвычайной стабильностью технологического процесса, контролируя ее с помощью индуктивных бесконтактных датчиков.

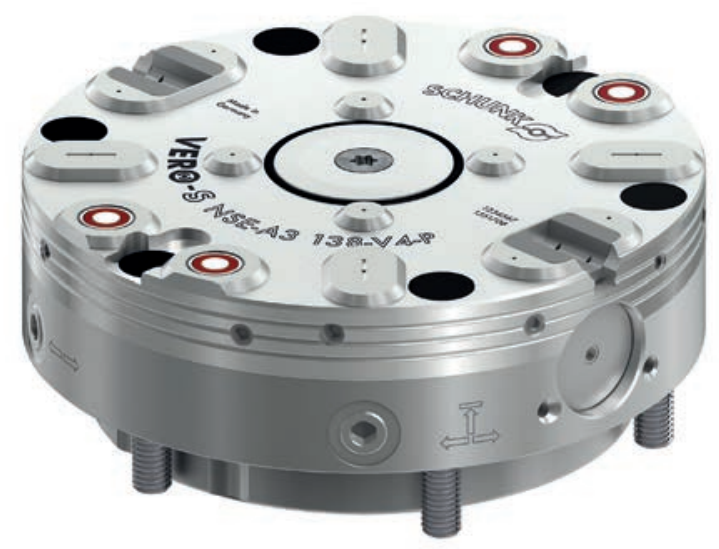

PUс. 6. Модульная система быстрой смены паллет VERO-S NSE-A3 138 была специально разработана для автоматизированных решений

\section{УСТРОЙСТВА АВТОМАТИЗАЦИИ СО ВСТРОЕННЫМ БЛОКОМ ПЕРЕДАЧИ СРЕДЫ}

Устройство автоматизации SCHUNK VERO-S NSE-A3 138 (рис. 6) продемонстрировало, что классические зажимные модули нулевой точки также можно оптимизировать для автоматических процессов. Для надежной смены заготовок и зажимных приспособлений устройство автоматизации оборудовано мощной функцией продувки, которая аккуратно очищает опорную поверхность в процессе смены. Кроме того, подпружиненное конусное уплотнение предотвращает проникновение стружки и грязи в интерфейс смены. Невероятное прижимное усилие, достигающее 8000 или $28000 \mathrm{H}$ при активированной функции «турбо», а также высокая стабильность размеров корпуса модуля дают преимущество в части жесткости технологических решений по автоматизированной быстрой смене. Благодаря этому можно эффективно компенсировать даже большие опрокидывающие моменты и срезающие усилия. Центрирующие вставки с гибкими элементами обеспечивают позиционную ориентацию с высокой повторяемостью, а также максимальную надежность автоматизированных процессов. С помощью конического фитинга прижимные пальцы невероятно легко подсоединяются к компоненту. Благодаря встроенному блоку передачи среды можно подавать жидкости с допустимым давлением системы до 300 бар (например, для контроля зажимных приспособлений или подачи компонентов для автоматического мониторинга).

\section{ТЕНДЕНЦИЯ К ПЕРЕХОДУ НА ЦИФРОВЫЕ ТЕХНОЛОГИИ}

Последние исследования технологий SCHUNK четко продемонстрировали курс движения: в допол- 
нение к механическим качествам зажимные приспособления будущего будут предоставлять все больше и больше цифровых возможностей (рис. 7). Например, «интеллектуальный» модуль для быстрой смены паллет SCHUNK VERO-S NSE-S3138 позволяет осуществлять постоянный мониторинг прижимного усилия и положения фиксирующего ползуна. Для этой цели компания SCHUNK предлагает тензометрические датчики, измерители усилия и индуктивные аналоговые датчики, встроенные непосредственно в модуль. Более того, присутствие заготовок или зажимных паллет можно определить с помощью индуктивных бесконтактных датчиков. Это обеспечивает оптимальные условия всего процесса зажатия. Несомненно, решающее преимущество «интеллектуальных» модулей заключается в их совместимости с программой чисто механических VERO-S: таким образом, вся интеллектуальная система была эффективно встроена в модульную зажимную систему, при этом размеры остались полностью идентичными размерам механических VERO-S NSE3. Сделав еще один шаг вперед в наших технологиях, мы создали силовой зажим- ной блок SCHUNK TANDEM KSE 100. В дополнение к встроенному мониторингу хода и усилия зажима, этот «интеллектуальный» силовой зажим также способен индивидуально регулировать усилие зажима и положение каждого кулачка на соответствующей заготовке. Это позволяет выполнить предварительное позиционирование зажимных кулачков в текущем процессе и сократить время цикла. Кроме того, можно уменьшить зажимное усилие для деталей, чувствительных к деформации, чтобы предотвратить их деформацию или повреждение поверхности. Необходимая сенсорная технология также полностью интегрирована в зажимной блок.

\section{МИХЕЛЬБЕРГЕР Маркус -}

глава отдела продаж систем зажима

\section{АУФРЕХТ Соня -}

дипломированный специалист по экономике предприятий (BA), журналист (FJS), менеджер по корпоративным коммуникациям и связям с общественностью

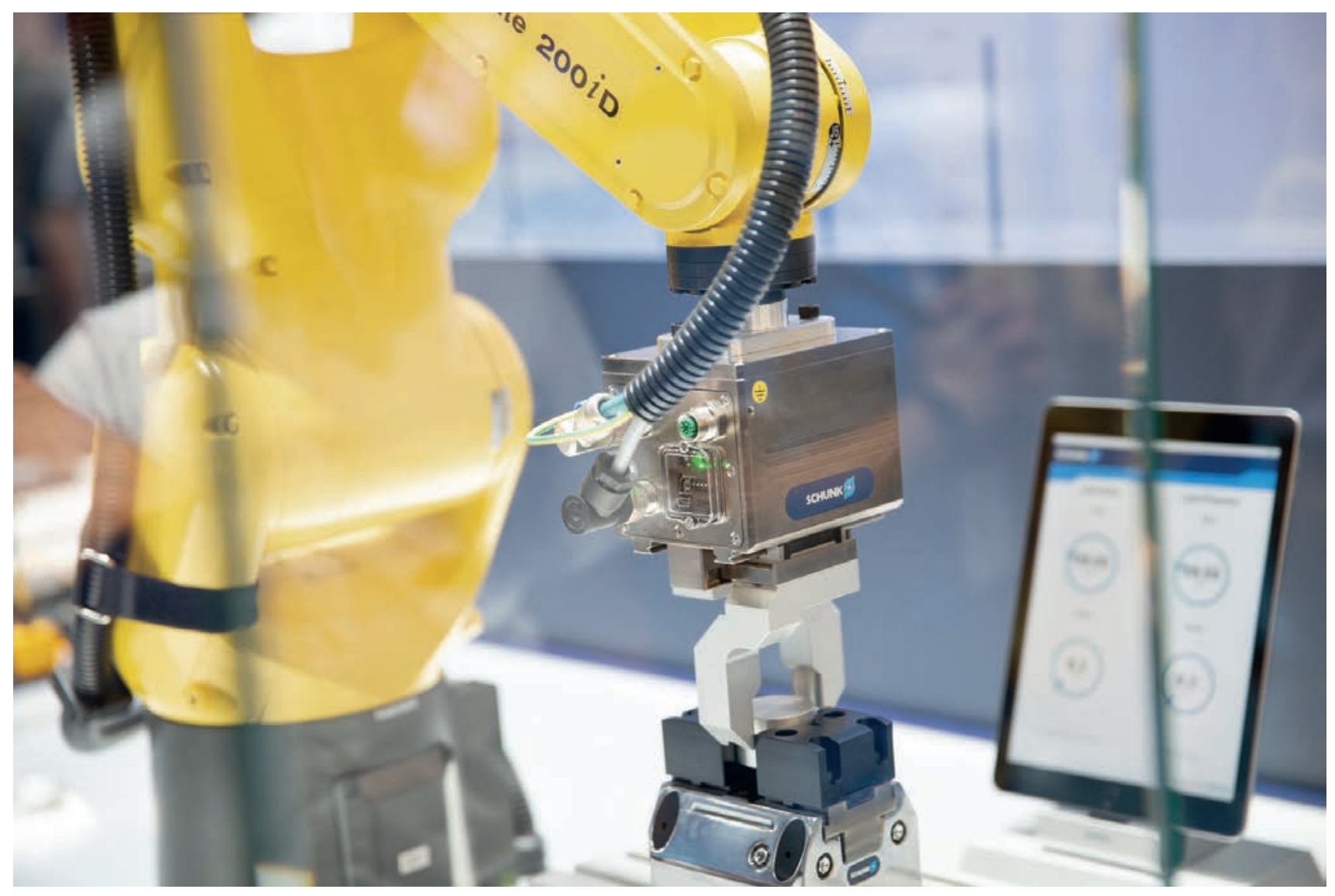

Puc. 7. «Умные» зажимные устройства и системы позволяют измерять, идентифицировать и контролировать зажатые компоненты и текущий производственный процесс в режиме реального времени 
